\title{
Paediatric Retinoblastoma in India: Evidence from the National Cancer Registry Programme
}

\author{
Sukanya Rangamani ${ }^{1}$, Krishnan SathishKumar ${ }^{1}$, N Manoharan ${ }^{2}$, Pramod Kumar \\ Julka $^{3}$, Goura Kishor Rath ${ }^{4}$, Viswanathan Shanta ${ }^{5}$, Rajaraman Swaminathan ${ }^{6}$, \\ Ranganathan Rama ${ }^{6}$, Karabi Datta ${ }^{7}$, Syamsundar Mandal $^{7}$, Shravani Koyande ${ }^{8}$, \\ Vinay Deshmane ${ }^{8}$, B Ganesh ${ }^{9}$, Shripad D Banavali ${ }^{10}$, Rajendra A Badwe ${ }^{11}$, C \\ Ramesh $^{12}$, Lingappa Appaji ${ }^{13}$, Ambakumar Nandakumar ${ }^{1 *}$
}

\begin{abstract}
Background: Globally, retinoblastoma is the most common primary intraocular malignancy occurring in children. This paper documents the recent incidence rates of retinoblastoma by age and sex groups from the Population Based Cancer Registries (PBCRs) of Bangalore, Mumbai, Chennai, Delhi and Kolkata using the data from the National Cancer Registry Programme. Materials and Methods: Relative proportions, sex ratio, method of diagnosis, and incidence rates (crude and age standardized) for each PBCR and pooled rates of the five PBCRs were calculated for the years 2005/06 to 2009/10. Standard errors and 95\% confidence limits of ASIRs by sex group in each PBCR were calculated using the Poisson distribution. Standardised rate ratios of ASIR by sex group and rate ratios at risk were also calculated. Results: The maximum retinoblastoma cases were in the $0-4$ age group, accounting for $78 \%$ (females) and $81 \%$ (males) of pooled cases from five PBCRs. The pooled crude incidence rate in the 0-14 age group was 3.5 and the pooled ASIR was 4.4 per million. The pooled ASIR in the 0-4, 5-9 and 10-14 age group were 9.6, 2.0 and 0.1 respectively. The $M / F$ ratio in Chennai (1.9) and Bangalore PBCRs (2.0) was much higher than the other PBCRs. Among the PBCRs, the highest incidence rate in 0-4 age group was found in males in Chennai (21.7 per million), and females in Kolkata (18.9 per million). There was a distinct variation in incidence rates in the PBCRs in different geographic regions of India.
\end{abstract}

Keywords: Retinoblastoma-population based cancer registry-incidence rate

Asian Pac J Cancer Prev, 16 (10), 4193-4198

\section{Introduction}

Globally, retinoblastoma is the most common primary intraocular malignancy occurring in children. The incidence of retinoblastoma reported in the Cancer Incidence in Five continents Volume $\mathrm{X}$ is lower in the developed nations as compared to the developing countries, and the age standardized incidence rates (ASIR) ranges from 1 per $1,000,000$ population in $0-14$ years male and female children in many parts of the world to 8 per 1,000,000 in Blantyre, Malawi in Africa (Forman et al.,2013). There have been earlier studies that have analysed the incidence of retinoblastoma from specific regions in India, that pertain to the last two decades
(Nandakumar et al., 1996; Yeole et al., 2002; Swaminathan et al., 2008). Retinoblastoma is a rare disease, and calculation of incidence rates requires reliable data from a defined population at risk. Using the data from the National Cancer Registry Programme (NCRP), India this paper explains the recent incidence rates of retinoblastoma by age and sex groups from the Population Based Cancer Registries (PBCRs) of Bangalore, Mumbai, Chennai, Delhi and Kolkata.

\section{Materials and Methods}

The NCRP in India was established in 1982 and has expanded to include 29 PBCRs till date. The NCRP

\footnotetext{
${ }^{I}$ National Centre for Disease Informatics and Research, Indian Council of Medical Research, ${ }^{12}$ Department of Epidemiology and Biostatistics, ${ }^{13}$ Department Paediatric Oncology, Kidwai Memorial Institute of Oncology, Bangalore, ${ }^{2}$ Delhi Cancer Registry, ${ }^{3}$ Department of Radiation Oncology, Delhi Cancer Registry, All India Institute of Medical Sciences, New Delhi, ${ }^{4}$ Department of Radiation Oncology, Dr.B.R.Ambedkar Institute Rotary Cancer Hospital, All India Institute of Medical Sciences, New Delhi, ${ }^{5}$ Directorate, ${ }^{6}$ Department of Biostatistics and Cancer Registries, Cancer Institute (WIA), Chennai, ${ }^{7}$ Department of Epidemiology and Biostatistics, Chittaranjan National Cancer Institute, Kolkata, ${ }^{8}$ Indian Cancer Society, ${ }^{9}$ Department of Biostatistics, Epidemiology and Cancer Registry, Tata Memorial Hospital, ${ }^{10}$ Department of Medical Oncology, ${ }^{11}$ Directorate, Tata Memorial Centre, Mumbai, India*For correspondence: ank@blr.vsnl.net.in
} 
collects information on incident cases of all cancers, diagnosed among residents (resident for at least a year or in children less than a year old, whose parents were resident for at least a year) in a defined population, from multiple sources and records it through a software data entry module. Information on deaths due to cancer are also collected from hospitals and the Civil registration system and matched with incident cases. Detection of duplicate registrations and internal validity checks are carried out by the software programme of the NCRP on an ongoing basis. The cancers are classified using ICD-O-3 (International Classification of diseases-Oncology, 3rd edition) morphology and topography codes and by anatomical site using ICD-10 (International Classification of Diseases10,10th revision). The NCRP produces periodic reports on ASIRs (standardized to world population), patterns of cancers, and time trends of incidence rates to explain the magnitude of cancer burden and highlight the priority areas of cancer research and control.

Cases registered with the respective PBCRs since its commencement [Mumbai, Chennai, Bangalore-1982, Delhi-1988, Kolkata-2005] till $31^{\text {st }}$ December of the last reported year (2010 for all registries and 2009 for Delhi and Kolkata) were extracted from the NCRP database. The inclusion criteria included all records with ICD-O-3 morphology codes of 9510 to 9513 . Variables of individual cases like name, age, sex, duration of stay at usual residence, date of first diagnosis, date of registration at the reporting institution, morphology, topography and sequence were extracted. Internal checks on the validity of the data were performed that included missing fields, age specified as $>14$ and consistency checks between topography and morphological coding. Records with discrepancies were corrected wherever possible by verifying the original records in the PBCRs. The latest completed data from 2006-2010 (Bangalore, Chennai, Mumbai) and 2005-2009 (Delhi, Kolkata) were used to calculate the incidence rates. Eight cases aged $>14$ years were excluded from analysis.
Population estimates in India are based on decadal national censuses conducted since 1961. Estimates of population by five yearly age groups have been projected based on difference distribution method for population projections (Takiar and Shobana, 2009). The 1991 and 2001 census were used for population estimates for the time period of 2006- 2010. Relative proportions, sex ratio, method of diagnosis, and incidence rates (crude and age standardized) for each PBCR and pooled rates of the five PBCRs were calculated for the years 2005/06 to 2009/10 to provide recent information. ASIRs was calculated by the direct method, using the world standard population, expressed per million population. Standard error and 95\% confidence limits of ASIRs by sex group in each PBCR were calculated using the Poisson approximation. Standardised Rate Ratio of ASIR by sex group with its $95 \%$ confidence limits was also determined. Rate Ratio at risk was calculated from cumulative risk and rate estimations.

Data from the Hospital based Cancer Registries (HBCRs) of Bangalore and Chennai (2006 to 2010), and Mumbai (2006-2008), were used to calculate relative proportions, sex ratio, and method of diagnosis.

\section{Results}

There were 214 registered retinoblastoma cases in age group 0-14 years from Bangalore, Chennai and Mumbai PBCRs (2006-2010) and Delhi and Kolkata PBCRs (20052009). Microscopic verification of histopathological diagnosis of retinoblastoma was available in $97.7 \%$ of pooled cases in PBCRs and $2.3 \%$ cases were diagnosed clinically or by other methods. In the HBCRs, microscopic verification of diagnosis $(77 \%)$, and xray and ultrasonogram $(23 \%)$ were the methods of diagnosis.

The maximum numbers were in the $0-4$ age group in both sexes, accounting for $78 \%$ (females) and $81 \%$ (males) of pooled cases from PBCRs. Children aged 5 -9 years accounted for 15 to $20 \%$ of cases, and thus, 98 to $100 \%$

Table 1A. Numbers, Relative Proportion by Age Group and Sex, Male Female Ratio of Retinoblastoma Cases in 0-14 Age Group from Selected PBCRs (2006-2010)

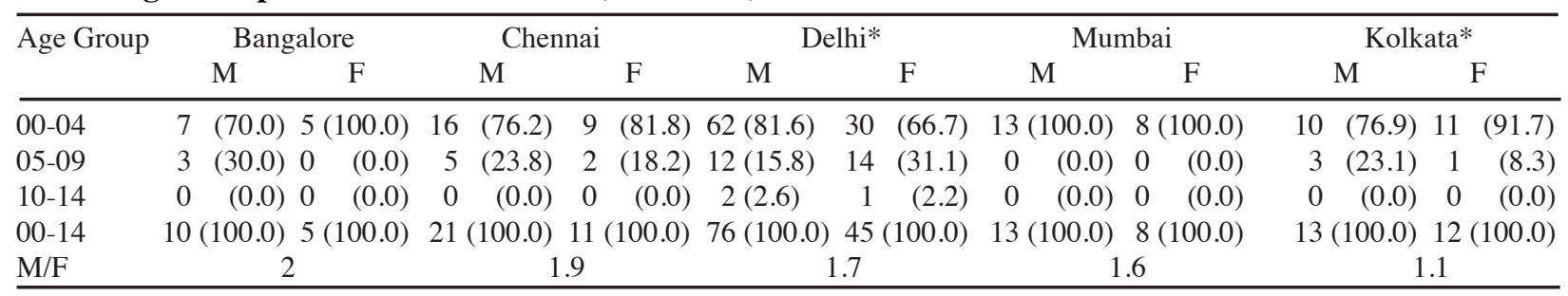

*Data 2005-2009

Table 1B. Numbers, Relative Proportion (\%) by Age Group and Sex, Male Female Ratio of Retinoblastoma Cases in 0-14 Age Group in Selected HBCRs (2006-2010)

\begin{tabular}{|c|c|c|c|c|c|c|}
\hline \multirow{3}{*}{$\begin{array}{l}\text { Age group } \\
00-04\end{array}$} & \multicolumn{2}{|c|}{ Bangalore } & \multicolumn{2}{|c|}{ Chennai } & \multicolumn{2}{|c|}{ Mumbai^ $^{\wedge}$} \\
\hline & M & $\mathrm{F}$ & $\mathrm{M}$ & $\mathrm{F}$ & $\mathrm{M}$ & $\mathrm{F}$ \\
\hline & $13(72.2)$ & $30(78.9)$ & $9 \quad(81.8)$ & $13(81.3)$ & $45 \quad(84.9)$ & $29(93.5)$ \\
\hline 05-09 & $5 \quad(27.8)$ & $8(21.1)$ & $2(18.2)$ & 3 (18.7) & $8 \quad(15.1)$ & $2 \quad(6.5)$ \\
\hline $10-14$ & $0 \quad(0.0)$ & $0 \quad(0.0)$ & $0 \quad(0.0)$ & $\begin{array}{ll}0 & (0.0)\end{array}$ & $(0.0)$ & $0 \quad(0.0)$ \\
\hline $00-14$ & $18(100.0)$ & $38(100.0)$ & $11(100.0)$ & $16(100.0)$ & $53(100.0)$ & $31(100.0)$ \\
\hline $\mathrm{M} / \mathrm{F}$ & \multicolumn{2}{|c|}{0.5} & \multicolumn{2}{|c|}{0.7} & \multicolumn{2}{|c|}{1.7} \\
\hline
\end{tabular}

^Data 2006-2008 
Paediatric Retinoblastoma in India: Evidence from the National Cancer Registry Programme

Table 2. Age Group Specific Incidence Rate, Crude Rate (0-14), Age Standardized (World) Incidence Rate(Per Million), Standard Error (SE), $95 \%$ Confidence Intervals of ASIR, Rate Ratio and $95 \%$ CI in Selected PBCRs (2006-2010)

\begin{tabular}{|c|c|c|c|c|c|c|c|c|c|c|c|c|c|}
\hline \multirow[t]{2}{*}{ Age group } & \multicolumn{2}{|c|}{ Bangalore } & \multicolumn{2}{|c|}{ Chennai } & \multicolumn{2}{|c|}{ Delhi* } & \multicolumn{2}{|c|}{ Mumbai } & \multicolumn{2}{|c|}{ Kolkata* } & \multicolumn{2}{|c|}{ Pooled } & \multirow[t]{2}{*}{ Both Sexes } \\
\hline & M & $\mathrm{F}$ & M & $\mathrm{F}$ & M & F & M & $\mathrm{F}$ & M & $\mathrm{F}$ & M & $\mathrm{F}$ & \\
\hline 00-04 & 5.1 & 3.9 & 21.7 & 12.3 & 15 & 8.6 & 5.2 & 3.4 & 15.9 & 18.9 & 11.5 & 7.5 & 9.6 \\
\hline 05-09 & 2.1 & 0 & 6.2 & 2.6 & 2.5 & 3.3 & 0 & 0 & 3.6 & 1.4 & 2.1 & 1.8 & 2.0 \\
\hline $10-14$ & 0 & 0 & 0 & 0 & 0.4 & 0.2 & 0 & 0 & 0 & 0 & 0.2 & 0.1 & 0.1 \\
\hline $00-14$ & 2.3 & 1.2 & 8.2 & 4.4 & 5.3 & 3.7 & 1.5 & 1 & 5.3 & 5.4 & 4.1 & 2.8 & 3.5 \\
\hline ASIR & 2.7 & 1.5 & 10.4 & 5.6 & 6.7 & 4.5 & 2 & 1.3 & 7.3 & 7.8 & 5.2 & 3.5 & 4.4 \\
\hline SE & 0.15 & 0.12 & 0.4 & 0.3 & 0.14 & 0.12 & 0.1 & 0.08 & 0.36 & 0.4 & 0.08 & 0.07 & 0.05 \\
\hline $95 \%$ CI & \multicolumn{2}{|c|}{$2.4-2.91 .3-1.7$} & \multicolumn{2}{|c|}{$9.6-11.25 .0-6.2$} & \multicolumn{2}{|c|}{$6.5-7.0 \quad 4.2-4.7$} & \multicolumn{2}{|c|}{$1.8-2.2 \quad 1.2-1.5$} & \multicolumn{2}{|c|}{$6.6-8.07 .0-8.5$} & \multicolumn{2}{|c|}{$5.0-5.3 \quad 3.4-3.6$} & $5 \quad 4.3-4.5$ \\
\hline Rate Ratio & \multicolumn{2}{|c|}{1.76} & \multicolumn{2}{|c|}{1.86} & \multicolumn{2}{|c|}{1.51} & \multicolumn{2}{|c|}{1.53} & \multicolumn{2}{|c|}{0.94} & \multicolumn{2}{|c|}{1.48} & \multirow{5}{*}{ 1:17070 } \\
\hline $95 \% \mathrm{CI}$ & \multicolumn{2}{|c|}{$1.46-2.11^{\#}$} & \multicolumn{2}{|c|}{$1.64-2.11^{\#}$} & \multicolumn{2}{|c|}{$1.42-1.61^{\#}$} & \multicolumn{2}{|c|}{$1.31-1.78^{\#}$} & \multicolumn{2}{|c|}{$0.82-1.08$} & \multicolumn{2}{|c|}{$1.41-1.55^{\#}$} & \\
\hline \multicolumn{3}{|l|}{ Ratio at Risk } & \multirow{3}{*}{\multicolumn{2}{|c|}{$\begin{array}{c}1: 7169 \\
1: 13423\end{array}$}} & \multirow{3}{*}{\multicolumn{2}{|c|}{$\begin{array}{l}1: 11174 \\
1: 16529\end{array}$}} & \multirow{3}{*}{\multicolumn{2}{|c|}{$\begin{array}{l}1: 38462 \\
1: 58824\end{array}$}} & \multirow{3}{*}{\multicolumn{2}{|c|}{$\begin{array}{c}1: 10257 \\
1: 9853\end{array}$}} & & & \\
\hline M & \multirow{2}{*}{\multicolumn{2}{|c|}{$1: 27758$}} & & & & & & & & & $1: 14$ & 497 & \\
\hline $\mathrm{F}$ & & & & & & & & & & & $1: 213$ & 311 & \\
\hline
\end{tabular}

Table 3. Comparative Incidence Rates (Crude and ASIR) of Retinoblastoma from Selected International Registries [Incidence Rate is Expressed Per Million Population]

\begin{tabular}{|c|c|c|c|c|c|c|}
\hline Region/Registry & $00-04$ & 05-09 & $10-14$ & $\begin{array}{c}00-14 \\
\mathrm{M}\end{array}$ & $\mathrm{F}$ & Total \\
\hline Thailand (2003-2005) & 7.6 & 0.5 & & & & 3.1 \\
\hline Korea (2006-2010) & 11.1 & 0.6 & & $5.6^{\wedge}$ & $5^{\wedge}$ & \\
\hline Great Britain [England,Scotland \&Wales] (1963-2002) & 10 & 0.4 & & 3.5 & 3.4 & 3.5 \\
\hline Australia $(1997-2006)$ & 11 & 0.6 & & 4.6 & 3.2 & 3.9 \\
\hline \multicolumn{7}{|l|}{ Canada (1992-2006) } \\
\hline$<1$ & 20.4 & 0.3 & 0.1 & 4 & 3.9 & 3.9 \\
\hline $1-4$ & 8.9 & & & & & \\
\hline USA-SEER (1975-2004) & 11.8 & & & & & \\
\hline India-pooled (2006-2010) CR & 9.6 & 2 & 0.1 & 4.1 & 2.8 & 3.5 \\
\hline India-pooled (2006-2010) ASIR & & & & 5.2 & 3.5 & 4.4 \\
\hline
\end{tabular}

$\wedge$ ASIR is for 0 to 9 years

cases occurred in 0-9 age group. The male to female (M/F) ratio varied from 1.1 (Kolkata) to 2.0 (Bangalore) (Table 1A). In the HBCRs, maximum numbers occurred in 0-4 age group (83\%), and the $\mathrm{M} / \mathrm{F}$ ratio was $<1$ in Bangalore and Chennai and 1.7 in Mumbai (Table 1B).

The pooled (all PBCRs) crude incidence rate in $0-14$ age group was 3.5 and the pooled ASIR was 4.4 per million. The pooled ASIR in the 0-4, 5-9 and 10-14 age group was 9.6, 2.0 and 0.1 respectively. The pooled ASIR in 0-14 age group by sex was 5.2 (males) and 3.5 (females). (Table 2).

The ASIR in 0-14 age group males ranged from 2 per million in Mumbai to 10.4 per million population in Chennai. In 0- 14 age group females, the ASIR ranged from 1.3 in Mumbai to 7.8 in Kolkata (Table 2). The ASIRs for males was higher than females in all PBCRs except Kolkata. The Rate Ratio of ASIR by sex was greater than 1 in all PBCRs $(\mathrm{p}<0.05)$ except Kolkata (Table 2$)$.

\section{Discussion}

Childhood cancers in age group 0-14 years ranged from $0.5 \%$ to $5.8 \%$ of all cancers in the PBCRs in India (National Cancer Registry Programme,2012). The four PBCRs and all the HBCRs have contributed to the NCRP since the 1980s except Kolkata PBCR (since 2005), and have established datasets for analysis with microscopically verified diagnosis of $97.7 \%$ in PBCRs and $77 \%$ in HBCRs. In both the PBCRs and HBCRs, the maximum numbers of cases occurred in the 0-4 age group, a pattern reported in earlier studies in India( Yeole et al., 2002; Swaminathan et al., 2008; Hazarika et al.,2014) and in developed nations (MacCarthy et al., 2009; Mitra et al., 2012). The M/F ratio in Chennai (1.9) and Bangalore PBCRs (2.0) was much higher than the other PBCRs. Globally the M/F ratio of retinoblastoma has been reported as unity,(Parkin et al.,1998; MacCarthy et al., 2009; Mitra et al., 2012) and in the past, Chennai had reported a M/F ratio of 1.0 (Swaminathan et al., 2008) and Mumbai of 1.4 (Yeole et al., 2002).

The pooled crude incidence rate ( 3.5 per million) and pooled ASIR (4.4 per million) in 0-14 age group, was similar to incidence rates reported in developed countries like Great Britain (MacCarthy et al., 2009), Canada (Mitra et al., 2012), Australia (Baade et al., 2010) and in Thailand (Wiangnon et al., 2011; 2014) (Table 3). Among the PBCRs, the highest incidence rate in 0-4 age group was found in males in Chennai (21.7 per million), and females in Kolkata (18.9 per million). The incidence rate in 0-4 age group males in Chennai (21.7) is the highest as compared to developing and developed nations (Table 3). Such similar high incidence rates have been reported only in age group below 1 year in Canada (20.9) (Mitra et al., 2012) and Great Britain (20.3) (MacCarthy et al., 2009). 
Retinoblastoma was among the top five childhood cancers reported by the Chennai PBCR(1990-2001) with ASIR of 7.6 (males) and 7.9 ( females) (Swaminathan et al., 2008). The latest data from the Population based Cancer Registries (2009-2011) in India indicate that ASIR of retinoblastoma could range from 6.7 per million population among 0-14 age group female children in Kolkata to 12.3 per million among $0-14$ age group male children in Chennai.

(National Cancer Registry Programme, 2012). In this study, Chennai had high rates in 0-14 males(10.4) and lower rate in females (5.6). The rates of Mumbai PBCR are two times lower than reported in the late 1980s (Yeole et al., 2002). The rates reported in Bangalore PBCR is lower than the incidence rate of 3.1 (both sexes) in 19821989 (Nandakumar et al., 1996). Kolkata is second to Chennai in incidence in males. The ASIR of 0-14 male children in Chennai(10.4) was higher than the maximum reported ASIR of microscopically verified retinoblastoma in Malawi, Africa (8.0 per million in males) in the latest release of Cancer Incidence in five continents Vol X (20032007) (Forman et al., 2013).

The incidence rates in both sexes were highest in the $0-4$ age group and decreased as age increased, being almost nil in 10-14 age group, a pattern observed globally (Table 3). The incidence rate in 0-4 age group, ranged from 5.1 (Bangalore) to 21.7 (Chennai) per million among males, and 3.4 (Mumbai) to 18.9 (Kolkata) among female children. The incidence rates in 0-4 male children were greater than the rates in female children in all PBCRs, except for Kolkata (Table 2). Previously, Mumbai PBCR had shown higher rates in 0-4 males (9.4) as compared to female children(5.9) (Yeole et al., 2002), while Chennai had revealed similar incidence rates in 0-4 male (15.9) and female (16.1) children (Swaminathan et al., 2008). Similar rates in both sexes in 0-4 age group has been reported from Great Britain and Korea (MacCarthy et al., 2009; Park et al.,2014). The Male Female Incidence Rate Ratio with $95 \%$ confidence limits (Table 2 ) revealed that the incidence rates were statistically significantly higher in males as compared to females when standardized to the world population in all PBCRs except Kolkata.

It was also observed that the trend in incidence rates in 0-4 years children (both sexes) showed a non -significant decrease over time in Bangalore, Chennai and Delhi, and statistically significant decrease in Mumbai (data not presented), which may be related to issues of low coverage. Trend analysis in developed nations show varied patterns- an increase in incidence in Great Britain (1983-2002) (MacCarthy et al., 2009), a decrease in incidence rates in Canada (1992-2006) among males (Mitra et al., 2012), and stable rates in the USA in both sexes (1975-2004) (Broaddus et al., 2009). In extremely rare cancers with low numbers, the trends observed may be due to random changes in the annual rates. Population expansion, changes in demographic composition and demarcation of geographic area, decrease in infant mortality rate, increased general awareness on cancer, improved diagnostic and treatment services, improved registration practices and case ascertainment, increase in reporting centres could be reasons for any apparent changes in incidence rates over time.

An increasing trend in incidence of unilateral cases in 0 to 4 years, especially among below 1 year children over 1963-2002 was reported in Great Britain. The authors postulated that this could be due to improved ascertainment of cases or a common exposure to an environmental factor (MacCarthy et al., 2009).Our present analysis revealed a distinct variation in incidence rates in the PBCRs of different geographic regions, though trends in incidence rates shown a non-significant decrease in three of the PBCRs. It is unknown if this variation in incidence between the PBCRs is related to environmental factors. Globally, risk factors like exposure to sunlight (Hooper, 1999; Jemal et al., 2000), viruses (Orjuela et al., 2000), low level radiation (Stiller, 1993) and parental dietary factors (Orjuela et al., 2005; Bunin et al.,2013) have been explored but the evidence has been inconclusive.

Retinoblastoma is a genetic disease with biallelic inactivation or loss of the retinoblastoma 1 gene-13q14.2. It is known to occur as heritable (mutations occurring in the germline and present in all somatic cells), and non -heritable or sporadic forms (with mutations arising locally within the developing retina). Sex differences have not been reported in the heritable or non-heritable forms. It is also known that all bilateral cases are heritable (26-40\%), and unilateral cases could be familial/heritable (10-15\%) and sporadic (50-70\%) (MacCarthy et al., 2009; Broaddus et al., 2009). On pooled analysis, 52.3\% cases were unilateral, $6.5 \%$ were reported as bilateral in the PBCRs, while in the HBCRs $71 \%$ were unilateral and $12.5 \%$ were bilateral cases. The Mumbai PBCR had reported $17 \%$ of bilateral cases in an earlier study(Yeole et al., 2002). Hospital based cancer registries in developing countries like Oman (Khandekar et al., 2004) and Saudi Arabia (Khandekar et al., 2014) (where consanguinity is well known), and Malaysia (Subramaniam et al., 2014) have reported $25-36 \%$ (bilateral) and 63-75\% (unilateral) retinoblastoma. Hospital based studies in India (Sahu et al., 1998 ; Harini et al., 2001) have reported consanguinity in $9-17 \%$ of children with retinoblastoma, an association that needs to be further studied.

The present study findings have to be interpreted carefully as they represent low number of cases over the latest five years of available data of a relatively rare childhood cancer. The database did not have complete information on laterality, sequence of eye affected, heritability, follow-up or survival, but has definitely opened up questions on trends in incidence, variation in age and sex group, and genotype-phenotype relationship.

It has be shown that children with positive family history of retinoblastoma who underwent regular comprehensive screening of their eyes since birth had early diagnosis (as young as 8 months), and treatment modalities that could save the eye, leading to better ocular and patient survival (Abramson et al., 2003). This aspect could be true of unilateral retinoblastoma cases as well if detected early. In a hospital based study of infants in Iran, the median age at diagnosis of retinoblastoma was 6.96 months and the overall 5 year survival was 77\% (Mehdiabadi GB et al.,2014), which was mainly attributed to early diagnosis and treatment interventions. 
Thus early detection needs to be emphasized through raising awareness of the medical fraternity (primary care physicians, obstetricians, pediatricians) to ask about family history of retinoblastoma, to conduct screening examination of the eye of the newborn and serially during a child's preventive health care visits, and facilitate prompt referral when there are risk factors like family history, leucokoria and strabismus. Public health programmes for raising awareness and screening of eyes of children as a component of existent child health programmes have to be considered, especially as $15 \%$ of cases were 5 to 9 years, which could indicate late diagnosis.

Current treatment modalities of systemic chemotherapy and focal treatment have contributed significantly to disability limitation and patient survival. There has been a considerable body of knowledge on the genetics of the retinoblastoma gene and its protein, and there is potential for developing targeted molecular therapies(Sachdeva and O'Brien, 2012). Survival studies of retinoblastoma have shown less than $50 \%$ absolute survival in Chennai PBCR (Swaminathan et al., 2008), suggesting that there is need for concerted efforts in screening, treatment and follow up of RB children with retinoblastoma in India. Genetic testing could inform the type of mutation (germline or somatic), risk of unilateral or bilateral disease, choice of treatment protocols and prognosis of the condition. A diagnostic model has been proposed for developing countries (Joseph et al., 2006), though genetic and clinical screening is still inaccessible and unaffordable for the affected children and families.

The retinoblastoma gene is a tumour suppressor gene and its mutations are related to the initiation and progression of cancers (Benedict et al., 1990). There is evidence that survivors of hereditary retinoblastoma are at increased risk as compared to non-heritable retinoblastoma cases, to develop non-ocular second primary tumors like sarcomas, brain tumour, melanoma, lung and bladder cancers(Moll et al.,1996; Kleinerman et al., 2000; 2007; Fletcher et al., 2007; MacCarthy et al., 2013). The risk is further increased if patients had been treated with radiotherapy for their retinoblastoma (Yu et al., 2009).

Projection of burden of retinoblastoma in India based on data of the NCRP revealed that there will be 1000 male and female children affected with retinoblastoma every year. As retinoblastoma is a unique disease due to its early age of occurrence, genetic aetiology, and risk of second cancers, there is tremendous scope for epidemiological and molecular genetics research.

\section{References}

Abramson DH, Beaverson K, Sangani P, et al (2003). Screening for retinoblastoma: presenting signs as prognosticators of patient and ocular survival. Pediatrics, 112, 1248-55.

Baade PD, Youlden DR, Valery PC, et al (2010). Trends in incidence of childhood cancer in Australia, 1983-2006. Br J Cancer, 102, 620-6.

Benedict WF, Xu HJ, Hu SX, Takahashi R (1990). Role of the retinoblastoma gene in the initiation and progression of human cancer. J Clin Investigat, 85, 988-93.

Broaddus E, Topham A, Singh AD (2009). Incidence of retinoblastoma in the USA: 1975-2004. Br J Ophthalmol,
93, 21-3.

Bunin GR, Li Y, Ganguly A, Meadows AT, Tseng M (2013). Parental nutrient intake and risk of retinoblastoma resulting from new germline RB1 mutation. Cancer Causes Control, 24, 343-55.

Forman D, Bray F, Brewster DH, et al (Eds) (2013).Cancer incidence in five continents, Vol. X (electronic version),IARC Lyon,. http://ci5.iarc.fr /CI5I-X/old/vol10/CI5-X-AllbyHisto.pdf, last accessed on [11 ${ }^{\text {th }}$ November, 2014].

Fletcher O, Easton D, Anderson K, et al (2004). Lifetime risks of common cancers among retinoblastoma survivors. J Natl Cancer Inst, 96, 357-63.

Harini R, Ata-ur-Rasheed M, Shanmugam MP, et al (2001) Genetic profile of 81 retinoblastoma patients from a referral hospital in southern India. Indian J Ophthalmol, 49, 37-42.

Hazarika M, Krishnatreya M, Bhuyan C,et al (2014). Overview of childhood cancers at a regional cancer centre in North-East India. Asian Pac J Cancer Prev, 15, 7817-9.

Hooper ML (1999). Is sunlight an aetiological agent in the genesis of retinoblastoma? Br J Cancer, 79, 1273-6.

Jemal A, Devesa SS, Fears TR, Fraumeni JF (2000). Retinoblastoma incidence and sunlight exposure. $\mathrm{Br} \mathrm{J}$ Cancer, 82, 1875-8.

Joseph B, Madhavan J, Mamatha G, et al (2006). Retinoblastoma: a diagnostic model for India. Asian Pac J Cancer Prev, 7,485-8.

Khandekar R, Ganesh A, Al Lawati J (2004). A 12-year epidemiological review of retinoblastoma in Omani children. Ophthalmic Epidemiol, 11, 151-9.

Khandekar RB, Al-Towerki AA, Al-Katan H, et al (2014). Ocular malignant tumors. review of the tumor registry at a tertiary eye hospital in central Saudi Arabia. Saudi Med $J, 35,377-84$.

Kleinerman RA, Tarone RE, Abramson DH,et al (2000). Hereditary retinoblastoma and risk of lung cancer. $J$ Natl Cancer Inst, 92, 2037-9.

Kleinerman RA, Tucker MA, Abramson DH, et al (2007). Risk of soft tissue sarcomas by individual subtype in survivors of hereditary retinoblastoma. J Natl Cancer Inst , 99, 24-31.

MacCarthy A,Birch JM, Draper GJ, et al, (2009). Retinoblastoma in Great Britain 1963-2002. Br J Ophthalmol, 93, 33-7.

MacCarthy A, Bayne AM, Brownbill PA, et al (2013). Second and subsequent tumours among 1927 retinoblastoma patients diagnosed in Britain 1951-2004. Br J Cancer, 108, 2455-63.

Mehdiabadi GB, Habibi R, Shariftabrizi A, Vossough P (2014). Epidemiologic survey of infantile cancer in Iran based on the data of the largest pediatric cancer referral center (AliAsghar Children Hospital), 1996-2005. Asian Pac J Cancer Prev, 15, 1211-7.

Mitra D, Shaw AK, Hutchings K (2012). Trends in incidence of childhood cancer in Canada,1992-2006. Chronic Dis Inj Can, 32, 131-9.

Moll AC, Imhof SM, Bouter LM, et al (1996). Second primary tumors in patients with hereditary retinoblastoma: a registerbased follow-up study, 1945-1994. Int J Cancer, 67, 515-9.

Nandakumar A, Anantha N, Appaji L, et al (1996). Descriptive epidemiology of childhood cancers in Bangalore, India. Cancer Causes Control, 7, 405-10.

National Cancer Registry Programme (ICMR) (2012). Three year report of population based cancer registries 2009-2011. Bangalore, India.

Orjuela M, Castaneda VP, Ridaura C, et al (2000). Presence of human papilloma virus in tumor tissue from children with retinoblastoma: an alternative mechanism for tumor development. Clin Cancer Res, 6, 4010-6.

Orjuela MA, Titievsky L, Liu X, et al (2005). Fruit and vegetable intake during pregnancy and risk for development of 

Prev, 14, 1433-40.

Park SJ, Woo SJ, Park KH (2014). Incidence of retinoblastoma and survival rate of retinoblastoma patients in Korea using the Korean National Cancer Registry database (1993-2010). Invest Ophthalmol Vis Sci, 55, 2816-21.

Parkin DM, Kramarova E, Draper GJ, (Eds) (1998). International incidence of childhood cancer vol. 2. IARC scientific publications No. 144, IARC, Lyon.

Sachdeva UM, O’Brien JM (2012). Understanding pRb: toward the necessary development of targeted treatments for retinoblastoma. J Clin Investigat, 122, 425-34.

Sahu S, Banavali SD, Pai SK, et al (1998). Retinoblastoma: problems and perspectives from India. Pediatr Hematol Oncol, 15, 501-8.

Stiller CA (1993). Retinoblastoma and low level radiation. BMJ, 307, 461-2.

Subramaniam S, Rahmat J, Rahman NA, et al (2014). Presentation of retinoblastoma patients in Malaysia. Asian Pac J Cancer Prev, 15, 7863-7.

Swaminathan R, Rama R, Shanta V (2008). Childhood cancers in Chennai, India, 1990-2001: incidence and survival. Int J Cancer, 122, 2607-11.

Takiar R, Shobana B (2009). Cancer incidence rates and the problem of denominators-a new approach in Indian cancer registries. Asian Pac J Cancer Prev, 10, 123-6.

Wiangnon S, Veerakul G, Nuchprayoon I, et al (2011). Childhood cancer incidence and survival 2003-2005, Thailand: study from the Thai pediatric oncology group. Asian Pac Cancer Prev, 12, 2215-20.

Wiangnon S, Jetsrisuparb A, Komvilaisak P, Suwanrungruang K (2014). Childhood cancer incidence and survival 19852009, Khon Kaen, Thailand. Asian Pac J Cancer Prev, 15, 7989-93.

Yeole BB, Advani S (2002). Retinoblastoma: an epidemiological appraisal with reference to a population in Mumbai, India. Asian Pac J Cancer Prev, 3, 17-21.

Yu C-L, Tucker MA, Abramson DH, et al (2009). Cause-specific mortality in long-term survivors of retinoblastoma. J Natl Cancer Inst, 101, 581-91. 